\title{
Research Progress on the Relationship between Vitamin D and Female Reproduction
}

\author{
Xiaofei $\mathrm{Xu}^{1-4}$, Yongxiu Hao ${ }^{1-4}$ and Jie Qiao ${ }^{1-6 *}$ \\ ${ }^{1}$ Center for Reproductive Medicine, Department of Obstetrics and Gynecology, Peking University Third Hospital, China \\ ${ }^{2}$ National Clinical Research Center for Obstetrics and Gynecology, China \\ ${ }^{3}$ Key Laboratory of Assisted Reproduction (Peking University), Ministry of Education, China \\ ${ }^{4}$ Beijing Key Laboratory of Reproductive Endocrinology and Assisted Reproductive Technology, B China \\ ${ }^{5}$ Beijing Advanced Innovation Center for Genomics, China \\ ${ }^{6}$ Research Units of Comprehensive Diagnosis and Treatment of Oocyte Maturation Arrest \\ *Corresponding author: Jie Qiao, Center for Reproductive Medicine, Department of Obstetrics and Gynecology, Peking University \\ Third Hospital, No.49 North Huayuan Road, Haidian District, Beijing 100191, China
}

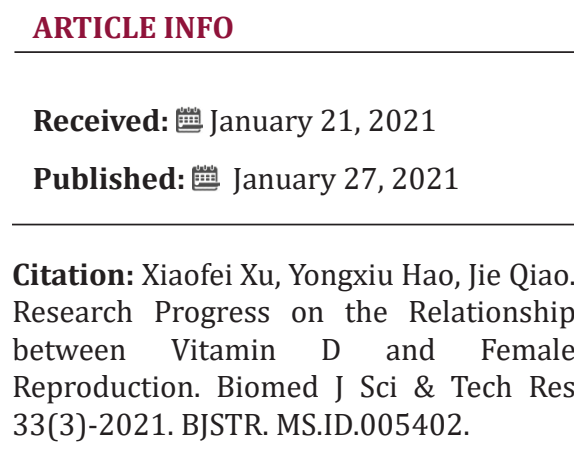

Keywords: Vitamin D; Female Reproduction; Anti-Mullerian Hormone; Polycystic Ovary Syndrome; In-vitro Fertilization; Pregnancy Outcome

\section{ABSTRACT}

Background: Vitamin D is a steroid hormone playing canonical roles in calcium metabolism and bone modeling. In recent years there has been a growing body of literature presenting associations between vitamin D levels and a variety of disease processes, including metabolic disorders, autoimmune conditions and reproductive system diseases.

Main Body: In females, there also has been many research exploring the role of vitamin D deficiency in polycystic ovary syndrome, in-vitro fertilization, pregnancy outcomes and so on. However, the results and studies evaluating the effects of replacing vitamin D have shown variable results. Therefore, we wrote this review to summarize the research progress about the potential role of vitamin $\mathrm{D}$ in female reproductive function and provide a perspective view for the following research.

Short Conclusion: There are controversial results of the studies. High quality RCTs and reliable fundamental studies are still needed to reach definitive conclusions.

List of Abbreviations: VDR: Vitamin D Receptor; PCOS: Polycystic Ovary Syndrome; AMH: Anti-Mullerian Hormone; IVF-ET: In-Vitro Fertilization-Embryo Transfer; ART: Assisted Reproductive Therapy; GDM: Gestational Diabetes; LGA: Large for Gestational Age

\section{Introduction}

Vitamin D is an essential steroid hormone playing crucial roles in human body with the main active forms of 25-(OH) D3, 1,25-(OH)2D3, 24,25-(OH)2D3 [1,2]. Clinically, the serum 25- $(\mathrm{OH})$ D3 concentration is usually measured to predict the vitamin D level. Vitamin D deficiency means a serum concentration of 25-(OH) D3 less than $20 \mathrm{ng} / \mathrm{mL}$, and vitamin D insufficiency means a 25-(OH) D3 level between $21 \mathrm{ng} / \mathrm{mL}$ and $29 \mathrm{ng} / \mathrm{Ml}$ [2]. The classical role of Vitamin D is maintaining the balance of calcium and phosphate homeostasis in intestines, bone, and the parathyroid glands. In recent years, Vitamin D receptor (VDR) expression has been confirmed in the other tissues besides the above classic target organs, such as immune cells, the pancreas, the cardiac system, the skeletal muscle. Moreover, VDR has been found in the female reproductive tract, such as ovary, uterus, fallopian tubes, placenta, etc. More recently there has been increasing interest in the role of vitamin $\mathrm{D}$ in reproductive function. 
Vitamin D deficiency is common in both the developed and developing countries [2]. In the United States, 20\%-90\% of reproductive-aged women have vitamin D deficient despite prenatal vitamin intake [2]. Numerous studies have explored the influences of vitamin D deficiency in polycystic ovary syndrome (PCOS), in-vitro fertilization, pregnancy outcomes and so on. However, the results and the effects of supplying vitamin D are still under debating. Therefore, we wrote this review to summarize the research progress about the potential role of vitamin D in female reproductive function and provide a perspective view for the following research.

\section{Vitamin D and AMH}

Anti-Mullerian hormone (AMH) is considered as an important marker of ovarian reserve used in clinical widely. One function of $\mathrm{AMH}$ is inhibiting recruitment of primordial follicles into folliculogenesis [3,4]. Epidemiology studies have shown an intricate correlation between vitamin D and AMH. One study enrolling thirtythree infertile women with normal ovarian reserve shown vitamin D altered AMH signaling and steroidogenesis in human cumulus GCs [5]. Another study recruiting 33 premenopausal women showed supplying vitamin D was able to reverse the seasonal decrease of AMH [6]. However, a retrospective cohort study reported that vitamin D levels were not associated with ovarian reserve in 457 infertile women (age: 21-50) with a high prevalence of diminished ovarian reserve [7].

And, a cross-sectional study in 73 healthy nonobese women of reproductive age without history of infertility did not find the correlation between vitamin D level and AMH too [8]. Moreover, a recent study demonstrated that patients with hereditary 1,25- $(\mathrm{OH}) 2 \mathrm{D}-$ resistant rickets who had a nonfunctioning VDR appeared to have a normal reproductive history, suggesting that partial effects of vitamin D on the reproductive system might be not direct [9]. Summarizing the above results suggests that there needs more prospective study with a large group as well as participants with similar backgrounds to confirm the correlation between vitamin D and $\mathrm{AMH}$

\section{Vitamin D and PCOS}

Polycystic ovary syndrome (PCOS) is the common endocrine disorder in women of reproductive age, and its prevalence is about $6-10 \%$ in general population. Moreover, PCOS is the major cause of anovulatory infertility, and is associated with insulin resistance (IR), hyperinsulinemia, dyslipidemia, and central obesity [10-13]. It is estimated $67 \%-85 \%$ of women with PCOS have vitamin D deficiency [14]. And there are plenty of studies related to vitamin D and PCOS. Studies suggested that there was an association between vitamin D status and hormonal as well as metabolic dysfunctions in PCOS [14], and they found that the active VDR complex regulated genes important for glucose and lipid metabolism [15]. However, some other studies did not support the results [16-21]. One study reported that Tehran women with and without PCOS had the similar vitamin D levels. Moreover, some intervention trials had been carried out to explore the effect of vitamin D supplementation.

Thys-Jacobs et al. [22] and Rashidi B et al. [23] found an improvement in menstrual regularity after vitamin D supplementation in women with PCOS. Selimoglu et al. [24] and Majid Dastorani et al. [25] observed a significant decrease in IR after the vitamin D supplementation, whereas the others failed to find a positive effect of vitamin D supplementation on IR [26-31]. Other than these above, some studies explored the correlation between VDR gene polymorphisms and PCOS. Ranjzad et al. found that the CASR 'TG' polymorphism was associated with IR [32]; Mahmoudi $\mathrm{T}$ found that the variants of VDR were associated with an increase in insulin levels and IR [33]; Wehr E et al. found that the VDR Apa-I genotype was associated with the prevalence of PCOS [34]. Nevertheless, Lin et al. [35], Zadeh-Vakili et al. [36] and Ranjzad F et al. [37] found no difference in VDR gene polymorphisms between PCOS and control women. Therefore, it is difficult to draw a definite conclusion for the relationship between vitamin D status and PCOS due to the heterogeneity of the studies. Well-designed prospective randomized clinical trials are still needed to investigate the relationship.

\section{Vitamin D and In-vitro Fertilization}

Whether vitamin D played a role in the process of in vitro fertilization are still elusive. Sebiha Ozkan et al. found that women with higher vitamin D level in serum and follicle fluid were more likely to achieve clinical pregnancy following in-vitro fertilizationembryo transfer (IVF-ET) [38]. A systematic review showed that there was a positive association between vitamin D status and assisted reproductive therapy (ART) outcomes and suggested vitamin D deficiency and insufficiency should be treated in women considering ARTs [39]. However, the majority of studies did not find a significant correlation between vitamin D level and IVF outcomes [40-42]. A meta-analysis concluded that there was not sufficient evidence supporting the necessary of vitamin D supplementation during IVF [43]. Thence, it still needs further analysis and exploration.

\section{Vitamin D and Pregnancy Outcomes}

Cumulative studies suggested that vitamin D deficiency related to adverse pregnancy outcomes, e.g. gestational diabetes (GDM), preeclampsia, pre-term birth and so on [44-49]. Recently, a systematic review indicated that pregnant women with low vitamin D level had an increased risk for GDM, and GDM could ameliorate after vitamin D supplementation [50]. A cohort study found that higher vitamin $\mathrm{D}$ ( $\geq 30 \mathrm{nmol} / \mathrm{L}$ ) in early pregnancy was associated with lower blood glucose both in early and throughout pregnancy, but higher vitamin D in late pregnancy was associated with higher risk of large for gestational age(LGA)at birth [51]. Interestingly, a recent research found that high vitamin $D$ at $15 \pm 1$ weeks' gestation 
was shown to be protective against the development of GDM and they also found that the correlation between vitamin D status and pregnancy complication might be was affected by fetal sex [52]. Mehri Jamilian et al. demonstrated that magnesium-zinc-calciumvitamin D co-supplementation to women with GDM might reduce biomarkers of inflammation and oxidative stress [53].

Sunni L Mumford and colleagues [54] presented increased rates of pregnancy and livebirth in women with sufficient serum concentrations of vitamin D preconception. Clinical trials about the effect of vitamin D supplementation during pregnancy shown conflicting results. Raden Tina Dewi Judistiani et al. suggested that sufficient maternal vitamin D was an important factor to improve fetal growth and development [55]. Hashemipour S et al., Roth DE et al. and Hossain $\mathrm{N}$ et al. found that treatment of low vitamin $\mathrm{D}$ during pregnancy improved the obstetric and neonatal outcomes [56-59]. Hornsby et al. demonstrated supplying vitamin D during pregnancy was beneficial for respiratory health in early life [60]. Urrutia-Pereira and Solé also reported low maternal vitamin D increased the risk for the later development of asthma and chronic obstructive pulmonary disease [61]. A post hoc analysis from RCT recommended regular exercise during pregnancy due to its positive influence on vitamin D level [62].

But Pérez-López FR et al. found that Vitamin D supplementation during pregnancy was not associated with maternal outcomes [63], and Moniek Looman et al. found vitamin D level or supplementation were not associated with glucose homeostasis in 105 women aged 18-40 years [64]. Some research explored the relationship between vitamin D level and postpartum depression, and the results were also perplexed [65-67]. Considering the heterogeneity of the above studies and the differences in vitamin D levels during pregnancy, non-pregnancy and different periods of pregnancy, larger, betterdesigned RCTs evaluating clinically relevant outcomes are necessary to reach a definitive conclusion.

\section{Conclusion}

The aim of this review was to present the findings of different kinds of studies on vitamin D and female reproduction. As presented, there are controversial results which could be due to the bias, inadequate power or other limitations of the studies. And the mechanisms are not clearly defined, whether vitamin D directly affecting female reproduction or via associated factors, such as AMH, hypocalcemia, insulin resistance, etc. playing indirect roles in reproductive outcomes is still confused. High quality RCTs are still needed to reach definitive conclusions, and reliable fundamental studies complement the mechanisms of vitamin D deficiency and supplementation in female reproduction ability and outcomes, ultimately set the appropriate vitamin D supplement doses for female.

\section{Authors' Contributions}

Xiaofei $\mathrm{Xu}$ was responsible for the search and selection of articles, extraction of data, and writing and revision of the manuscript. Jie Qiao initiated the study, contributed to the study design, and revised the paper critically for important content. Yongxiu Hao revised the paper critically. All the authors were involved in the final approval of the version to be published.

\section{References}

1. Henry HL NA (1984) Vitamin D: metabolism and biological actions. Annu Rev Nutr 4: 493-520.

2. Holick MF, Robert P Heaney, M Hassan Murad, Connie M Weaver, Endocrine Society, et al. (2011) Evaluation, treatment, and prevention of vitamin D deficiency: An Endocrine Society clinical practice guideline. J Clin Endocrinol Metab 96(7): 1911-1930.

3. Jeppesen JV (2013) Which follicles make the most anti-Mullerian hormone in humans? Evidence for an abrupt decline in AMH production at the time of follicle selection. Mol Hum Reprod 19(8): 519-527.

4. Wang H (2011) Inhibitory Actions of Anti-Müllerian Hormone (AMH) on Ovarian Primordial Follicle Assembly. PLoS ONE.

5. Merhi Z (2014) Vitamin D alters genes involved in follicular development and steroidogenesis in human cumulus granulosa cells. J Clin Endocrinol Metab 99(6): E1137-E1145.

6. Dennis NA (2012) The level of serum anti-Mullerian hormone correlates with vitamin D status in men and women but not in boys. J Clin Endocrinol Metab 97(7): 2450-2455.

7. Shapiro AJ (2018) Vitamin D levels are not associated with ovarian reserve in a group of infertile women with a high prevalance of diminished ovarian reserve. Fertil Steril 110(4): 761-766.

8. Chang EM (2014) Association between sex steroids, ovarian reserve, and vitamin D levels in healthy nonobese women. J Clin Endocrinol Metab 99(7): 2526-2532.

9. Tiosano D, Weisman Y (2019) Reproductive history of patients with hereditary 1,25-dihydroxyvitamin D-resistant rickets. Fertility and Sterility 112(1): 156-161.

10. de Groot (2011) PCOS, coronary heart disease, stroke and the influence of obesity: a systematic review and meta-analysis. Hum Reprod Update 17(4): 495-500.

11. Diamanti Kandarakis E, A Dunaif (2012) Insulin resistance and the polycystic ovary syndrome revisited: an update on mechanisms and implications. Endocr Rev 33(6): 981-1030.

12. Wild RA, Manfredi Rizzo, Sheri Clifton, Enrico Carmina (2011) Lipid levels in polycystic ovary syndrome: systematic review and metaanalysis. Fertil Steril 95(3): 1073.

13. Sirmans SM, KA Pate (2013) Epidemiology, diagnosis, and management of polycystic ovary syndrome. Clin Epidemiol 6: 1-13.

14. Krul Poel YHM (2018) Vitamin D and metabolic disturbances in polycystic ovary syndrome (PCOS): A cross-sectional study. PLoS One 13(12): e0204748.

15. Bouillon R, Hilary F Luderer, Liesbet Lieben, Chantal Mathieu, Marie Demay, et al. (2008) Vitamin D and human health: lessons from vitamin D receptor null mice. Endocr Rev29(6): 726-776.

16. Hahn S, S Roesler, R Kimmig, K Mann, O E Janssen, et al. (2006) Low serum 25-hydroxyvitamin D concentrations are associated with insulin resistance and obesity in women with polycystic ovary syndrome. Exp Clin Endocrinol Diabetes 114(10): 577-583.

17. Li HW (2011) Vitamin D deficiency is common and associated with metabolic risk factors in patients with polycystic ovary syndrome. Metabolism 60(10): 1475-1481.

18. Ngo DT (2011) Determinants of insulin responsiveness in young women: Impact of polycystic ovarian syndrome, nitric oxide, and vitamin D. Nitric Oxide 25(3): 326-330. 
19. Wehr E, A Giuliani, D Kopera, T R Pieber, B Obermayer Pietsch, et al. (2009) Association of hypovitaminosis D with metabolic disturbances in polycystic ovary syndrome. Eur J Endocrinol 161(4): 575-582.

20. Yildizhan R (2009) Serum 25-hydroxyvitamin D concentrations in obese and non-obese women with polycystic ovary syndrome. Arch Gynecol Obstet 280(4): 559-563.

21. Patra SK (2012) Vitamin D as a predictor of insulin resistance in polycystic ovarian syndrome. Diabetes Metab Syndr 6(3): 146-149.

22. SusanThys Jacobs D, AnatasioPapadopoulos, PhilipSarrel, John PBilezikian (1999) Vitamin D and calcium dysregulation in the polycystic ovarian syndrome. Steroids 64(6): 430-435.

23. Batool Rashidi, FH, Mamak Shariat, Farid Zayerii (2009) The Effects of Calcium-Vitamin D and Metformin on Polycystic Ovary Syndrome: A Pilot Study. Taiwanese Journal of Obstetrics and Gynecology 48(2): 142147.

24. Selimoglu H, G Ozkaya, E Tuncel, E Erturk, S Imamoglu, et al. (2010) The effect of vitamin D replacement therapy on insulin resistance and androgen levels in women with polycystic ovary syndrome. J Endocrinol Invest 33(4): 234-238.

25. Dastorani M (2018) The effects of vitamin D supplementation on metabolic profiles and gene expression of insulin and lipid metabolism in infertile polycystic ovary syndrome candidates for in vitro fertilization. Reprod Biol Endocrinol 16(1): 94.

26. Ardabili HR, BP Gargari, L Farzadi (2012) Vitamin D supplementation has no effect on insulin resistance assessment in women with polycystic ovary syndrome and vitamin D deficiency. Nutr Res 32(3): 195-201.

27. Kotsa K (2009) Role of vitamin D treatment in glucose metabolism in polycystic ovary syndrome. Fertil Steril 92(3): 1053-1058.

28. Pal L (2012) Therapeutic implications of vitamin D and calcium in overweight women with polycystic ovary syndrome. Gynecol Endocrinol 28(12): 965-968

29. Wehr E, TR Pieber, B Obermayer Pietsch (2011) Effect of vitamin D3 treatment on glucose metabolism and menstrual frequency in polycystic ovary syndrome women: a pilot study. J Endocrinol Invest 34(10): 757 63.

30. Shokoufeh Bonakdaran, ZMK, Behrooz Davachi, Javad Mazloom Khorasani (2012) The effects of calcitriol on improvement of insulin resistance, ovulation and comparison with metformin therapy in PCOS patients: a randomized placebo- controlled clinical trial. Iran J Reprod Med 10(5): 465-472.

31. Sathyapalan T, Charlotte Arnett, Anne-Marie Coady, Eric S Kilpatrick, Stephen L Atkin, et al. (2010) Atorvastatin increases 25-hydroxy vitamin D concentrations in patients with polycystic ovary syndrome. Clin Chem 56(11): 1696-700.

32. Ranjzad F, Touraj Mahmoudi, Mohsen Vahedi, Abdolrahim Nikzamir, Mohammad Reza Zali, et al. (2011) Influence of gene variants related to calcium homeostasis on biochemical parameters of women with polycystic ovary syndrome. J Assist Reprod Genet 28(3): 225-232.

33. Mahmoudi T (2009) Genetic variation in the vitamin D receptor and polycystic ovary syndrome risk. Fertil Steril 92(4): 1381-1383.

34. Wehr E (2011) Vitamin D-associated polymorphisms are related to insulin resistance and vitamin D deficiency in polycystic ovary syndrome. European Journal of Endocrinology 164(5): 741-749.

35. Lin MW (2012) Vitamin D receptor 1a promotor -1521 G/C and -1012 A/G polymorphisms in polycystic ovary syndrome. Taiwan J Obstet Gynecol 51(4): 565-571.

36. Zadeh Vakili A (2013) Genetic polymorphism of vitamin D receptor gene affects the phenotype of PCOS. Gene 515(1): 193-196.

37. Ranjzad F (2012) A common variant in the adiponectin gene and polycystic ovary syndrome risk. Mol Biol Rep 39(3): 2313-2319.
38. Ozkan S, Jun Shu, Gohar Zeitlian, Cheryl Hickmon, Lubna Pal, et al (2010) Replete vitamin D stores predict reproductive success following in vitro fertilization. Fertil Steril 94(4): 1314-1319.

39. Chu J (2018) Vitamin D and assisted reproductive treatment outcome: a systematic review and meta-analysis. Hum Reprod 33(1): 65-80.

40. Firouzabadi RD (2014) Value of follicular fluid vitamin D in predicting the pregnancy rate in an IVF program. Arch Gynecol Obstet 289(1): 201206.

41. Aleyasin A (2011) Predictive value of the level of vitamin D in follicular fluid on the outcome of assisted reproductive technology. Eur J Obstet Gynecol Reprod Biol 159(1): 132-137.

42. Franasiak JM, Eric J Forman, Marie D Werner, Kathleen H Hong, Richard T Scott, et al. (2015) Vitamin D levels do not affect IVF outcomes following the transfer of euploid blastocysts. Am J Obstet Gynecol 212(3): 315.

43. Vanni VS (2014) Somigliana E, Papaleo E, Paffoni A, Pagliardini L, Candiani M., Vitamin D and assisted reproduction technologies: current concepts. Reprod Biol Endocrinol.

44. Wei S (2014) Obstetrics, and Gynecology, Vitamin D and pregnancy outcomes. Curr Opin Obstet Gynecol 26(6): 438-447.

45. Lewis S (2010) Vitamin D deficiency and pregnancy: from preconception to birth. Mol Nutr Food Res 54(8): 1092-1102.

46. Harvey NC (2014) Vitamin D supplementation in pregnancy: a systematic review. Health Technol Assess 18(45): 1-190.

47. Aghajafari, F, Paul E Ronksley, Suzanne C Tough, Maeve O’Beirne, Doreen M Rabi, et al. (2013) Association between maternal serum 25-hydroxyvitamin D level and pregnancy and neonatal outcomes: systematic review and meta-analysis of observational studies. BMJ 346: f1169.

48. Pashapour S (2019) Relationship between low maternal vitamin D status and the risk of severe preeclampsia: A case control study. Pregnancy Hypertension 15: 161-165.

49. Hong Bi S, Yang Xiaowu, Wang Ying, Xu Yang, Cao Ting, et al. (2018) High prevalence of vitamin D deficiency in pregnant women and its relationship with adverse pregnancy outcomes in Guizhou, China. J Int Med Res 46(11): 4500-4505.

50. Robinson CJ (2018) Vitamin D: a possible intervention for preventing gestational diabetes? BJOG 125(7): 794.

51. Walsh M, Bärebring L, Augustin H (2019) Avoiding maternal vitamin D deficiency may lower blood glucose in pregnancy. The Journal of Steroid Biochemistry and Molecular Biology 186: 117-121.

52. Wilson RL (2018) Vitamin D levels in an Australian and New Zealand cohort and the association with pregnancy outcome. BMC Pregnancy Childbirth 18(1): 251.

53. Jamilian M, Fereshteh Bahmani, Maryam Shokrpour, Maryam Chamani, Zatollah Asemi, et al. (2019) The effects of magnesium-zinc-calciumvitamin D co-supplementation on biomarkers of inflammation, oxidative stress and pregnancy outcomes in gestational diabetes. BMC Pregnancy Childbirth 19(1): 107.

54. Mumford SL (2018) Association of preconception serum 25-hydroxyvitamin D concentrations with livebirth and pregnancy loss: a prospective cohort study. The Lancet Diabetes \& Endocrinology 6(9): 725-732.

55. Judistiani RTD, Mohammad Ghozali, Yunia Sribudiani, Tetty Yuniati, Rizky Abdulah, et al. (2019) Association of first trimester maternal vitamin D, ferritin and hemoglobin level with third trimester fetal biometry: result from cohort study on vitamin D status and its impact during pregnancy and childhood in Indonesia. BMC Pregnancy Childbirth 19(1): 112.

56. Hashemipour S (2014) Effect of treatment of vitamin D deficiency and insufficiency during pregnancy on fetal growth indices and maternal weight gain: a randomized clinical trial. Eur J Obstet Gynecol Reprod Biol 172: 15-19. 
57. Roth DE (2013) Maternal vitamin D3 supplementation during the third trimester of pregnancy: effects on infant growth in a longitudinal followup study in Bangladesh. J Pediatr 163(6): 1605-1611.

58. Hossain N, Robina Kausar, Shabana Ayaz, Rafiq Khanani, Lubna Pal, et al. (2014) Obstetric and neonatal outcomes of maternal vitamin D supplementation: results of an open label, randomized controlled trial of antenatal vitamin D supplementation in Pakistani women. J Clin Endocrinol Metab 99(7): 2448-55.

59. Chakhtoura M (2016) Effect of vitamin D replacement on maternal and neonatal outcomes: a randomised controlled trial in pregnant women with hypovitaminosis D. A protocol .BMJ Open 6(3): e010818.

60. Hornsby E, Scott T Weiss, Vincent J Carey, George O'Connor, Catherine Hawrylowicz, et al. (2018) Vitamin D supplementation during pregnancy: Effect on the neonatal immune system in a randomized controlled trial. J Allergy Clin Immunol 141(1): 269-278.

61. Urrutia Pereira M, D Solé (2015) Vitamin D deficiency in pregnancy and its impact on the fetus, the newborn and in childhood. Revista Paulista de Pediatria (English Edition) 33(1): 104-113

62. Gustafsson MK, Mats Peder Mosti, Unni Syversen, Kjell Åsmund Salvesen, Per Medbøe Thorsby, et al. (2019) The effect of an exercise program in pregnancy on vitamin D status among healthy, pregnant Norwegian

\section{ISSN: 2574-1241}

DOI: $10.26717 /$ BJSTR.2021.33.005402

Jie Qiao. Biomed J Sci \& Tech Res

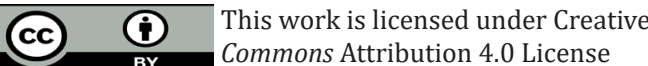

Submission Link: https://biomedres.us/submit-manuscript.php women: a randomized controlled trial. BMC Pregnancy Childbirth 19(1): 76.

63. Perez-Lopez FR (2015) Effect of vitamin D supplementation during pregnancy on maternal and neonatal outcomes: a systematic review and meta-analysis of randomized controlled trials. Fertil Steril 103(5): $1278-88 \mathrm{e} 4$

64. Looman M (2019) Changes in Micronutrient Intake and Status, Diet Quality and Glucose Tolerance from Preconception to the Second Trimester of Pregnancy. Nutrients 11(2): 460.

65. Abedi P (2018) The Relationship Between Vitamin D and Postpartum Depression in Reproductive-Aged Iranian Women. J Med Life 11(4): 286292.

66. Nielsen NO, Marika Lundqvist, Arieh Cohen, David M Hougaard, Mads Melbye, et al. 2013) Vitamin D Status during Pregnancy and the Risk of Subsequent Postpartum Depression: A Case-Control Study 8(11): e80686.

67. Wang J, Weijia Sun, Dandan Chen, Jingxia Zhao, Wei Zhang, et al. (2018) Association between vitamin D deficiency and antepartum and postpartum depression: a systematic review and meta-analysis of longitudinal studies. Arch Gynecol Obstet 298(6): 1045-1059.

$\begin{array}{ll}\text { BIOMEDICAL } & \text { Assets of Publishing with us } \\ \text { RESEARCHES } & \text { - Global archiving of articles } \\ \text { - Immediate, unrestricted online access } & \text { - Rigorous Peer Review Process } \\ & \text { - Authors Retain Copyrights }\end{array}$

